\title{
MORTALIDADE POR CHIKUNGUNYA NO ESTADO DA BAHIA NOS ANOS DE 2014 A 2017
}

\section{Iago Barbosa Ribeiro ${ }^{1}$; Erenilde Marques de Cerquira² ${ }^{2}$ e Hortência Lima}

\section{Almeida $^{3}$}

1. Bolsista PROBIC, Graduando em Enfermagem, Universidade Estadual de Feira de Santana, e-mail: iagobarbosa04@gmail.com.

2. Orientador, Departamento de Saúde, Universidade Estadual de Feira de Santana, e-mail: eremarques@fsonline.com.br

3. Participante do NUPEVS, Graduanda em enfermagem, Departamento de Saúde, Universidade Estadual de Feira de Santana, e-mail: hort.enfer@outlook.com

PALAVRAS-CHAVE: Chikungunya, Vigilância epidemiológica, Infecções por Arbovírus

\section{INTRODUÇÃO}

As arboviroses são caracterizadas por um grupo de doenças virais, transmitidas por vetores (Arthropod-borne vírus). Estas têm sido reconhecidas pela Organização Mundial de Saúde (OMS) como um problema global de saúde pública, em virtude de sua crescente dispersão territorial e necessidade de ações de prevenção e controle cada vez mais complexas (WHO, 2009).

A chikungunya é uma delas, acredita-se que procedência africana, onde o vírus circula enzooticamente entre primatas e pequenos animais, tendo se expandido para as zonas urbanas resultando em grandes epidemias (DIALLO et al, 1999; BROOK et al 2004).

A doença possui um período de incubação de dois a doze dias, e pode manifestar-se clinicamente de três formas: aguda, subaguda e crônica. Na fase aguda, os sintomas são muito parecidos com aqueles da dengue, exceto a característica e a intensidade do comprometimento nas articulações, mais frequente nas extremidades e grandes articulações; essa artrite/artralgia geralmente simétrica e intensa, podendo apresentar edema associado à rigidez e à limitação de movimento. Os sinais e sintomas aparecem de forma brusca com febre alta de início súbito, cefaleia, mialgia e artralgia. É frequente a ocorrência de manifestações cutâneas como o exantema maculopapular precoce, bem como lesões bolhosas entre outras. As dores nas articulações podem durar meses ou anos e, em certos casos, evoluir para dor crônica incapacitante que pode durar até cinco anos (BORGHERINI; et. al, 2007; SIMON; et al.2007; THIBERVILLE; et al.,2013.)

A doença no Brasil mostrou um caráter explosivo, por conta disso, diversas pessoas adoeceram concomitantemente e apresentando quadro clínico de grande severidade. As ações de enfrentamento envolveram profissionais de saúde e pesquisadores de várias partes do País em uma rede de cooperação e apoio às ações municipais, estaduais e federais com vistas ao enfrentamento da epidemia.

Em setembro de 2014 foram confirmados surtos da doença no município de Oiapoque (AP) e em Feira de Santana (BA), já com a transmissão autóctone do vírus nesses municípios (FEIRA DE SANTANA, 2014). 
O ano de 2016 houve uma segunda epidemia, que apresentou aumento no número de casos, pode ser explicado por dois fatores, o primeiro, envolve a população majoritariamente virgem em termos imunológicos em relação ao vírus do chikungunya, e o segundo fator são os índices elevados de infestação do Aedes aegypti (BRASIL, 2016).

$\mathrm{Na}$ edição $\mathrm{n}^{\circ} 174$ da Radis (2017) destaca que devido ao surto de febre amarela, fez a chikungunya perdesse a importância, hoje negligenciada pela mídia, os números de casos registados chegaram a 271.824 no ano de 2016, sendo o Nordeste com maior incidência, alcançando $86 \%$ das ocorrências. Outro porto que deve ser tomado em consideração é o aumento do número de óbitos pela doença que subiu de 14 em 2015 para 196 em 2016.

O objetivo do trabalho é analisar os indicadores de mortalidade por chikungunya no estado da Bahia nos anos de 2014 a 2017

\section{MATERIAL E MÉTODOS OU METODOLOGIA (ou equivalente)}

Trata-se de um estudo ecológico de série temporal com abordagem quantitativa, foram utilizados os dados do período de 2014 a 2017 obtidos na Superintendência de Proteção da Saúde (SUVISA) da Secretária de Saúde do Estado da Bahia (SESAB) e do Sistema de Informação de Mortalidade (SIM). A coleta ocorreu no mês junho de 2018. A análise e processamento dos dados foi feita a partir do Programa Microsoft Office Excel, versão 2016. Utilizando coeficientes e proporções, sendo estes dados apresentados em gráficos e tabelas.

\section{RESULTADOS E/OU DISCUSSÃO}

A figura 1 mostra o coeficiente de mortalidade por Chikungunya, evidencia que no início da epidemia que aconteceu em 2014 a população que foi afligida pela doença apresentou maior risco de morte em comparação com os anos seguintes.

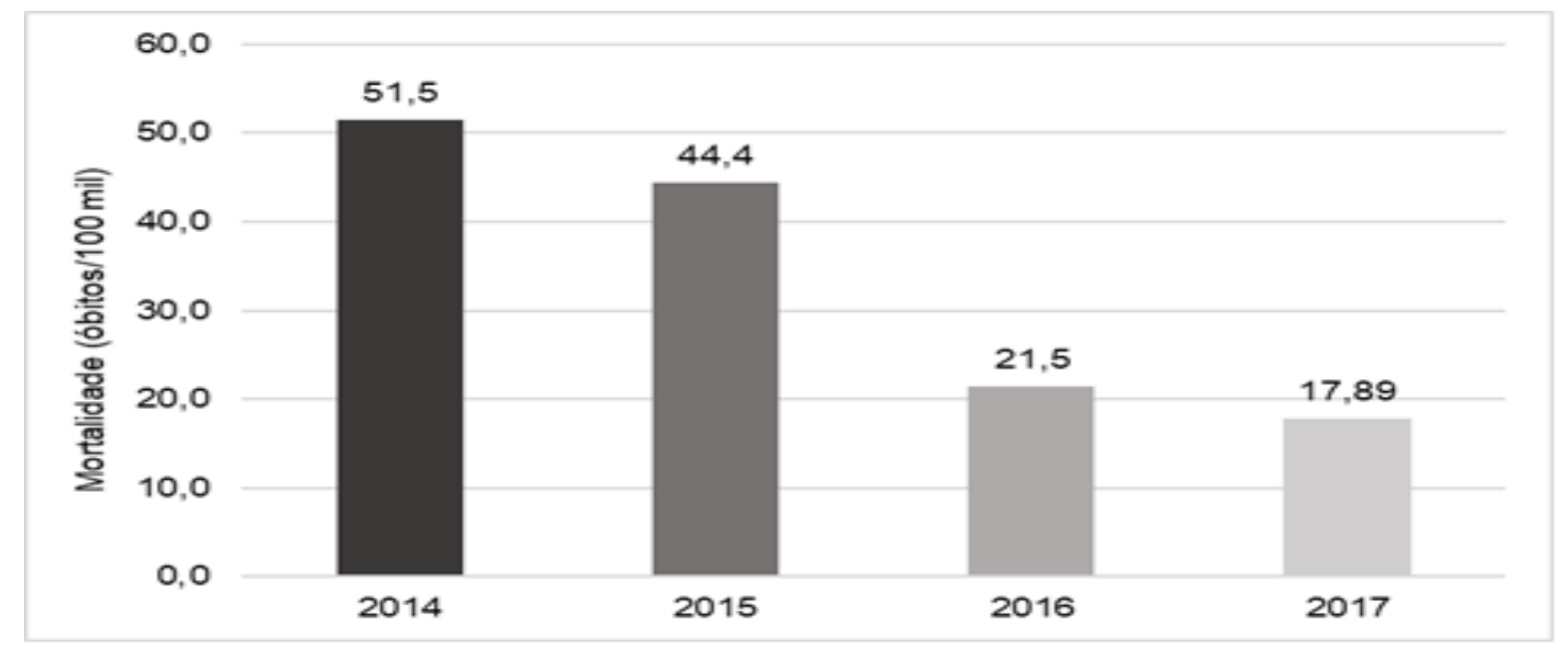

Figura 1- Coeficiente de mortalidade por Chikunguya no estado da Bahia nos anos de 2014 a 2017 Fonte: SUVISA/SESAB//DIS/SIM 
Observa-se que a taxa sobrevivente é $99,96 \%$ enquanto a taxa de óbitos por Chikungunya equivale a $0,04 \%$ dos casos notificados entre os anos de 2014 a 2017 no estado da Bahia. Mesmo que a doença não apresentando altas taxas de mortalidade, traz consigo a fase crônica da doença, que marcada artralgia inflamatória nas mesmas articulações afetadas durante os estágios agudos, que por sua vez interfere na qualidade de vida dos pacientes acometidos pela doença.

Novos estudos devem ser desenvolvidos para analisar a percepção dos indivíduos sobre a doença e como esta repercute na sua qualidade de vida. Os resultados do estudo demonstram a necessidade de melhorias no setor da vigilância epidemiológica, sendo necessário o fortalecimento e intensificação das suas ações, a capacitação profissional e as ações de educação em saúde para a conscientização da população acerca dos focos de transmissão do mosquito, contudo sabe-se que por conta das ações governamentais estão preconizando as ações e vigilância da Saúde.

\section{REFERÊNCIAS}

BROOK G; BETEL IS; MORSE AS. Humanarboviralinfection. In: Jawetz, MelnickandAdelberg's Medical Microbiology. 23rd. Singapore: Mc Graw Hill, p.514- 24. 2004.

DIALLO M; THONNON J; TRAORELAMIZANA M; FONTENILLE D. Vector of Chikungunya vírus in Senegal:current data andtransmissioncycles. Am J TropMedHyg. 60: p.281-6. 1999.

BORGHERINI, G. et al. Outbreak of Chikungunya on Reunion Island: Early Clinical and Laborato-ry Features in 157 Adult Patients. Clinical Infectious Diseases, v.44, p.1401-1407, 2007.

SIMON, F. et al. Chikungunya infection: an emerging rheumatism among travelers returned from Indian Ocean islands. Report of 47 cases. Medicine (Baltimore), v.86, p.123-137, 2007. THIBERVILLE, S.D. et al. Chikungunya fever: epidemiology, clinical syndrome, pathogenesis and therapy. Antiviral Research, v.99, n.3, p.345-370, 2013.

FEIRA DE SANTANA. Secretaria Municipal de Saúde. Boletim da Febre do Chikungunya. Situação epidemiológica dos casos de chikungunya. 2014; Edição 03, de 06 de outubro de 2014.

BRASIL. Boletim epidemiológico- Secretaria de Vigilância em Saúde. Ministério da Saúde, v14 (3), 2016. Monitoramento dos casos de dengue, febre chikungunya e febre pelo vírus Zika até a Semana Epidemiológica 13, 2016.

PERREIRA, M.G. Epidemiologia: teoria e prática. -[Reimpr.]. - Rio de Janeiro: Guanabara Koogan, 2012. p.596.

LIMA, JRC, PORDEUS, AMJ. ROUQUAYROL, MZ. Medida da Saúde Coletiva. Cap. 3, p. 42 - 64. In: Rouquayrol, MZ \& Gurgel, M (ORG). Rouquayrol - Epidemiologia e saúde. MedBook. $7^{\mathrm{a}}$ edição, 2013.

WORLD HEALTH ORGANIZATION. Dengue: Guidelines for treatment, prevention and control. Geneva: World Health Organization. WHO Library Cataloguing-in-Publication Data. New Edititon, 2009. Disponível em: http://www.who.int/tdr/publications/documents/denguediagnosis.pdf. 